\title{
69. Variation of the Water Content of Leaves in Relation to the Wilting of Plants."
}

\author{
By Rïchiro KôKetsu. \\ Botanical Institute, Kyushu Imperial University. \\ (Comm. by M. Mryoshi, M.I.A., April 12, 1928.)
}

It is a well known fact, that the water content of leaves decreases progressively, as a plant wilts. Moreover, it is suggested that any definite degree of wilting in a tissue would be found to correspond to a definite water deficit in the tissue, because the wilting of plants is nothing else than a symptom of water deficiency in the tissues. CALDWELL $^{2)}$ discovered that a plant has a definite amount of water in the tissues, when the wilting begins and at the time of so-called permanent wilting. But our knowledge of this relation is at present quite insufficient. The present paper is to report the results of the writer's work, which was undertaken to bring some contributions to the solution of this problem.

The plant materials used were Coleus Blumei, Glycine Soja and Mimosa pudica, all planted in pots. For the cultivation of the plants several kinds of soil or soil mixture with different water holding capacity were employed in order to study the influence of soil nature upon the relation in question. For the determination of water content, its percentage on the basis of both fresh and dry weight of material, and its value per unit area of the leaf were calculated. But it was found as expected, that the values calculated by the area method were preferable to those calculated by the percentage methods in our case. The values found by this method principally were adopted for the study of the results of experiments. When Mimosa was used for experiment, however, the values found by the dry weight method came primarily into consideration, because the area method was not applicable for this material.

According to the results of experiments the plants used contained a definite amount of water in the leaf tissue at the critical point of wilting (BRIGGS and SHANTZ' point of permanent wilting, or KôKETSU's critical point of wilting of Mimosa $)^{3}$. But the value of the water

1) Contribution from the Botanical Laboratory, Kyushu Imperial University.

2) J. S. Caldwell, Physiol. Research 1: 1-56, 1913.

3) L. J. Briggs and H. L. Shantz, Bot. Gaz. 53: 20-37 and 229-235, 1912.

R. Kôketsu, Bot. Mag. ('l'okyo) 39: 152-158, 1925. 
content was affected not a little by the culture conditions. The higher the water holding capacity of culture soils, the larger was the critical water content of leaves of Glycine, while the results found in Coleus were vice versa. The reason for these reversed phenomena is not immediately apparent. If, however, we assume that a mesophytic plant, such as Coleus, has smaller power to deprive the soil of moisture, while a semixerophytic plant, such as Glycine, has larger power, then a plant such as Coleus will fall into the critical state of wilting and will contain less moisture in the leaves, when it is cultivated in a soil with higher water holding capacity but with higher resistance against the water depriving power of plant, while the circumstances for a plant such as Glycine will be reversed. The phenomenon in question, therefore, seems to have some meaning relative to the xerophytic nature of plants.

The water content of leaves at the critical point of wilting proved to be very different according to the difference of plants. The ratio of this critical water content to the content at the stage of full turgidity was also differed widely. The values found in the individuals of Glycine cultivated in five different kinds of soil fluctuated between $0.51-0.74$ with the average 0.61 in an experiment series and between $0.51-0.73$ with the average 0.59 in another series, while the values found in Coleus cultivated in four different soils gave a fluctuation between $0.89-0.94$ with the average 0.91 . The value in question found in Mimosa plants, which were cultivated in but one kind of soil, was 0.53 . The value of this ratio was found, therefore, to be much higher in Coleus than in the other two materials, while the values for these latter two were near to each other. This ratio or the relation between the water content at the time of critical wilting and of full turgidity is probably specific to a given plant, even if the influence of the difference of its external conditions act not a little upon this relation, the values of this ratio showing the degree of the resisting power of a plant to wilting; an index to compare the grade of the xerophytism of plants, the more xerophytic a plant is, the lower is this value. 